\title{
Educación alimentaria y nutricional para reducir la obesidad en escolares de Panamá: protocolo de estudio
}

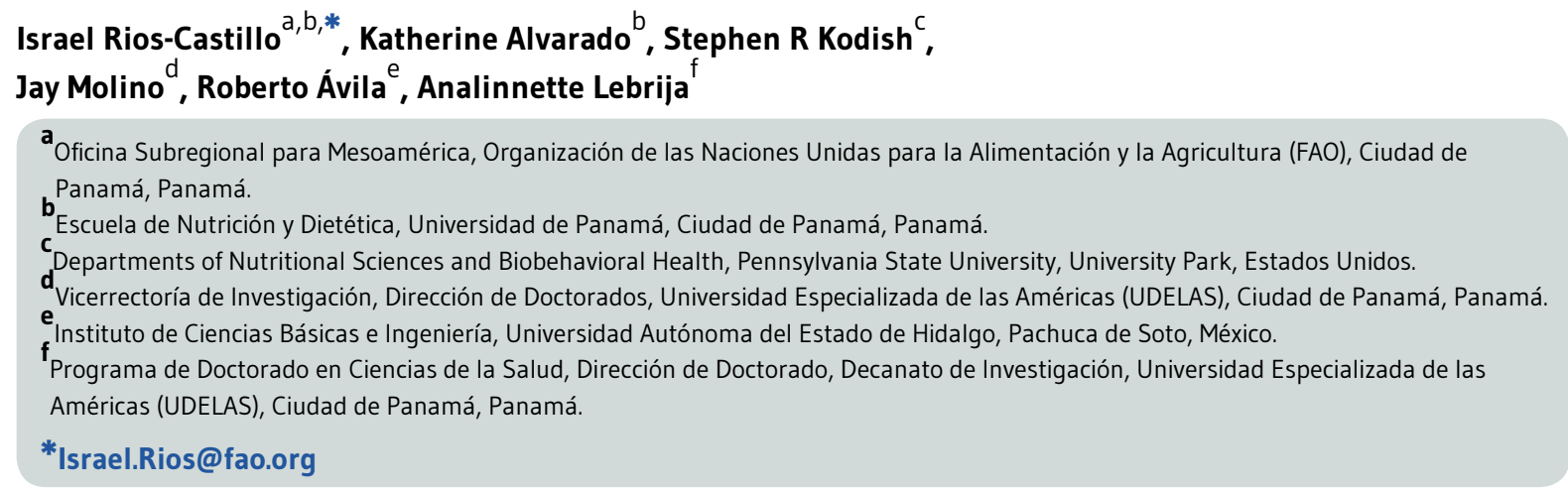

Editor Asignado: Eduard Baladia. Comité Editorial de la Revista Española de Nutrición Humana y Dietética. Pamplona, España.

Recibido el 1 de abril de 2019; aceptado el 29 de diciembre de 2019; publicado el 19 de enero de 2020.

Educación alimentaria y nutricional para reducir la obesidad en escolares de Panamá: protocolo de estudio

\section{PALABRAS CLAVE}

Sobrepeso;

Obesidad;

Nutrición;

Educación Alimentaria y Nutricional.

\section{RESUMEN}

El sobrepeso en escolares representa un creciente problema de salud pública en Panamá. El objetivo es desarrollar y validar un programa de intervención en educación alimentaria y nutricional (EAN) contra el sobrepeso y la obesidad en escolares de Panamá bajo la perspectiva de una política pública intersectorial de salud y educación. La intervención se basa en el cambio de conducta bajo el modelo socio-ecológico que favorezca la adopción de comportamientos alimentarios y de estilos de vida más saludables. Se trata de un estudio en 362 escolares de diseño cuasi experimental, pre-post intervención, prospectivo y sin grupo control, de tipo observacional explicativo en instituciones de educación básica general del sector público en tres Distritos de Panamá Oeste: La Chorrera, Capira y Chame. Se seleccionaron 5 escuelas: la escuela Victoria D'Spinay y La Herradura en el Distrito de La Chorrera, la escuela Hernando Bárcenas en el Distrito de Capira y las escuelas El Espavé y Berta Elida Fernández en el Distrito de Chame. El protocolo fue registrado en la plataforma internacional de ensayos № ISRCTN28920505. Se obtendrán datos sociodemográficos, antropométricos, nutricionales y alimentarios; de seguridad alimentaria; y de conocimiento, actitud, percepción y comportamiento sobre alimentación y estilos de vida saludables de marzo a diciembre de 2019 durante la implementación del programa intersectorial denominado "Escuelas Saludables" del Ministerio de Salud y del Ministerio de Educación de Panamá. Se describirán y compararán los datos utilizando estadística paramétrica y no paramétrica. El protocolo de estudio fue revisado y aprobado por el Comité de Bioética de la Universidad de Panamá (Ref. CBUP/№ 075/2019), registrado en la Dirección General de Salud (DIGESA) del MINSA (№ 881). Se solicitará consentimiento informado firmado por parte de los acudientes y asentimiento por cada niño participante. Los resultados del estudio serán difundidos ampliamente en revistas indexadas de revisión de pares y en congresos de salud y nutrición. 
Food and nutrition education to reduce obesity in schoolchildren in Panama: study protocol

KEYWORDS

Overweight;

Obesity;

Nutrition;

Food and Nutrition

Education.

\section{ABSTRACT}

Overweight in school-age children represents a growing public health problem in Panama. The objective is to develop and validate an intervention program in food and nutrition education (FNE) against overweight and obesity in school-age children in Panama from the perspective of an intersectoral public policy in health and education. The intervention is based on behavior changes under the socio-ecological model that favors the adoption of healthier dietary practices and lifestyles. This is a study in 362 school-age children of quasi-experimental, pre-test and post-test, prospective and non-controlled design, in public education institutions in three districts of Panama West Province: La Chorrera, Capira and Chame. Five schools were selected: the Victoria D'Spinay and La Herradura schools in the District of La Chorrera, the Hernando Bárcenas school in the District of Capira and the El Espavé and Berta Elida Fernández schools in the Chame District. The protocol was registered in the international test platform N ${ }^{\circ}$ ISRCTN28920505. Sociodemographic, anthropometric, nutritional and food data will be obtained; of food security; and of knowledge, attitude, perception and behavior on food and healthy lifestyles from March to December 2019 during the implementation of the intersectoral program called "Healthy Schools" of the Ministry of Health and the Ministry of Education of Panama. The data will be described and compared using parametric and non-parametric statistics. The study protocol was reviewed and approved by the Bioethics Committee of the University of Panama (Ref. CBUP/№075/2019), registered in the General Directorate of Health (DIGESA) of the Ministry of Health (№ 881). Signed informed consent will be requested from the guardians and assent for each participating child. The results of the study will be widely disseminated in peer-reviewed journals and in health and nutrition congresses.

\section{CITA}

Rios-Castillo I, Alvarado K, Kodish SR, Molino J, Ávila R, Lebrija A. Educación alimentaria y nutricional para reducir la obesidad en escolares de Panamá: protocolo de estudio. Rev Esp Nutr Hum Diet. 2020; 24(1): 78-86. doi: 10.14306/renhyd.24.1.776

\section{INTRODUCCIÓN}

El exceso de peso afecta a uno de cada tres estudiantes de escuelas primarias de Panamá1,2. El exceso de peso en la infancia es un importante factor de riesgo para el desarrollo de la obesidad en la adultez ${ }^{3,4}$. Asimismo, la obesidad es un factor de riesgo para el desarrollo de las enfermedades crónicas no transmisibles (ENT) que incluyen la diabetes, la hipertensión arterial, las enfermedades cardiovasculares y el cáncer ${ }^{5-8}$. Por su parte, el ambiente obesogénico en la escuela; la disponibilidad y el acceso a alimentos poco saludables; y la baja actividad física influyen en los hábitos alimenticios y estado nutricional de los escolares.

En Panamá se están implementando cambios en el ambiente escolar para contrarrestar la situación de malnutrición del país; éstos incluyen el establecimiento y regulación de los kioscos y cafetines escolares; la disposición de opciones saludables de meriendas; el incremento de la actividad física; cambios de conductas hacia prácticas alimentarias más sanas; y, acciones de educación alimentaria y nutricional (EAN). Así como, la provisión de agua segura, programas de alimentación escolar saludable y culturalmente aceptable, entre otras acciones. Todas estas intervenciones han sido recomendadas como políticas públicas efectivas para reducir y controlar el exceso de peso entre niños de edad escolar de primaria $^{9-13}$

El programa intersectorial de "Escuelas Saludables" (ES) está actualmente bajo implementación como estudio piloto en un número reducido de escuelas del sistema público de educación en Panamá, y es parte de las políticas de salud del Ministerio de Salud (MINSA) ${ }^{14}$. Dicho programa promueve la realización de actividad física, acciones de EAN, la regulación de venta de productos procesados y ultraprocesados, 
de bebidas azucaradas en las escuelas, además de la provisión de agua segura. Las ES se implementa articuladamente a través de comités locales incluyendo la participación del MINSA, Ministerio de Educación (MEDUCA) y el Ministerio de Desarrollo Agropecuario (MIDA).

A pesar de los esfuerzos realizados, la evidencia sugiere que las prácticas alimentarias poco saludables y la malnutrición son comunes entre los niños de edad escolar de primaria, sus padres y maestros. En Panamá, por ejemplo, se ha reportado elevado consumo de alimentos con alto contenido de grasas, sodio y azúcar en niños de edad preescolar; además, un alto consumo de bebidas azucaradas y de productos procesados y ultraprocesados en las meriendas de escolares y adolescentes cursando la educación media15,16.

Por otra parte, influir en el cambio de actitud a través de la EAN que facilite la adopción de comportamientos alimentarios y de estilos de vida saludables de manera sostenible es un desafío para prevenir el exceso de peso en escolares $^{17,18}$. Para ello, es necesario estudiar las bases conceptuales del problema; la actitud individual y colectiva hacia él; los mecanismos psicológicos y educativos más efectivos; y los modelos teóricos de cambio de comportamientos en salud para readaptar las conductas alimentarias y de estilos de vida que contribuyan al funcionamiento biológico y social de los individuos ${ }^{19-21}$.

Las intervenciones de EAN, por lo tanto, requieren ser integrales y empleando enfoques intersectoriales que consideren acciones no sólo a nivel individual, sino que además busquen la modificación de los ambientes alimentarios y de los sistemas alimentarios $22-24$. Dado el limitado número de evaluaciones de políticas públicas contra el exceso de peso entre niños de edad escolar en países en vías de desarrollo, se requieren intervenciones basadas en evidencia para escalarlas a políticas nacionales que puedan reducir y prevenir de manera efectiva el sobrepeso y la obesidad en este grupo de edad. El objetivo del presente protocolo es elaborar una alternativa metodológica de EAN para la prevención del sobrepeso y la obesidad en escolares (8-12 años) de Panamá. Este artículo describe el protocolo para los resultados primarios del estudio.

\section{MATERIAL Y MÉTODOS}

\section{Diseño de estudio}

Se trata de un estudio de diseño cuasi experimental pre y post intervención, prospectivo y sin grupo control de tipo observacional, explicativo para validar la implementación de un programa de EAN contra el sobrepeso y la obesidad en niños de edad escolar de escuelas públicas de Panamá. El levantamiento de la línea de base será en abril de 2019. El seguimiento se realizará a las 34 semanas, durante el año escolar 2019. Los hallazgos de este estudio serán reportados de acuerdo con los estándares establecidos en CONSORT para ensayos experimentales ${ }^{25}$.

\section{Población y selección de la muestra}

La población de estudio está conformada por niños de ambos sexos en edad escolar del sistema público de educación de Panamá en la Provincia de Panamá Oeste, que corresponden a instituciones educativas bajo la supervisión del MEDUCA. El estudio será realizado en cinco escuelas, Victoria D'Spinay y La Herradura en el Distrito de La Chorrera; la escuela Hernando Bárcenas en el Distrito de Capira; y, las escuelas de El Espavé y Berta Elida Fernández en el Distrito de Chame.

Las escuelas de La Herradura, Hernando Bárcenas y Berta Elida forman parte del programa intersectorial denominado ES, en donde se busca que los alumnos logren un adecuado estado de salud física, mental y social, y crear un ambiente de bienestar institucional y comunal a través de la promoción de estilos de vida saludable. En el proyecto ES participan además las familias, los maestros, el personal de la escuela y la comunidad. Este proyecto favorece el aprendizaje de los escolares, fortaleciendo el programa de salud escolar del MINSA, capacitando a maestros con conocimiento y habilidades necesarias para lograr cambios de actitud y prácticas dirigidas a mejorar el desarrollo integral de los estudiantes. Las dos escuelas restantes, Victoria D'Spinay y El Espavé fueron seleccionadas por sugerencias de los equipos de investigación del MINSA y MEDUCA con la finalidad de comparar resultados de ES en el estado nutricional.

La muestra del estudio estimada es de 302 sujetos de 8 a 12 años (cuarto a sexto grado). Se considerará un $10 \%$ adicional por posibles pérdidas en el seguimiento, por tanto, se trabajará con una muestra de 362 estudiantes. Se seleccionarán estudiantes desde cuarto grado debido a la facilidad de entrevistas y porque los contenidos sobre higiene, alimentación y nutrición se encuentran integrados en la currícula a partir de dicho nivel.

El tipo de muestreo a utilizar es por conveniencia, incluyendo aquellos estudiantes que estén disponibles durante el período de evaluación en coordinación con los directores y maestros de grado. El cálculo del tamaño de la muestra se realizó utilizando la fórmula para la estimación de 
una proporción poblacional tomando como referencia el dato reportado de sobrepeso por la Encuesta de Niveles de Vida (ENV) de 2008 para escolares de 5-9 años de edad de $26,9 \%{ }^{1}$. Los criterios empleados en el cálculo de la muestra fueron confidencialidad del $95 \%$ y precisión del $5 \%$.

El análisis de los datos se realizará únicamente con sujetos que cumplan con todas las evaluaciones en los períodos pre y post intervención. En caso de obtener una pérdida mayor del $20 \%$ se realizará un análisis de ingresados versus sujetos que dejaron de participar, utilizando los datos de la medición basal. Para minimizar las pérdidas se utilizará una ficha de registro para cada estudiante que incluya información de ubicación física. Sin embargo, el riesgo de deserción es mínimo debido a que el estudio se realizará durante las jornadas escolares.

\section{Criterios de inclusión y exclusión}

Se incluirán estudiantes de ambos sexos en edades comprendidas de 8 a 12 años (cuarto grado a sexto grado). Se excluirán aquellos sujetos que presenten discapacidad física/motora; que presenten enfermedad crónica diagnosticada (por ejemplo, diabetes) también se excluirán niños que presenten condiciones tales como parálisis cerebral, discapacidad cognitiva u otra condición que impida la realización de las medidas antropométricas y de las entrevistas.

Las variables respuestas primarias y secundarias del estudio se medirán al inicio y a las 34 semanas de clases. Para evaluar el estado nutricional se obtendrá el puntaje $z$ de índice de masa corporal para la edad (zIMCe), la circunferencia de la cintura y el porcentaje de grasa corporal. En base a las medidas antropométricas se estimará la proporción de niños con sobrepeso (zIMCe >1 DE) y la proporción de niños con obesidad (zIMCe >2 DE).

Se medirán además el nivel de conocimiento sobre la alimentación saludable en base a las Guías Alimentarias Basadas en Alimentos de Panamá (GABA) ${ }^{26}$; se evaluará la actitud, la percepción y el comportamiento hacia enunciados de prácticas dietéticas y de estilos de vida saludables utilizando un instrumento validado en 2018 con 51 ítems ${ }^{27}$. Las variables respuestas secundarias incluyen la ingesta dietética de macro y micronutrientes a través de recordatorio de 24 horas; la inseguridad alimentaria en el hogar mediante escalas de experiencias ${ }^{28}$ y la diversidad alimentaria utilizando el índice de diversidad dietética del hogar e individual ${ }^{29}$. Además, se realizarán entrevistas con estudiantes, padres, directores, maestros y personal administrativo para evaluar la aceptabilidad de las intervenciones.

\section{Procedimientos}

Evaluación sociodemográfica: Se empleará una encuesta sociodemográfica para obtener datos generales de los participantes, del hogar y de sus padres. El instrumento desarrollado es inédito y se ha sometido a validación de contenido por jueces pares y a dos estudios pilotos ( $\mathrm{n} 1=30$ y $\mathrm{n} 2=248$ ) para evaluar su validez y confiabilidad. Los pilotos se realizaron en agosto y septiembre de 2018 con estudiantes de las localidades elegidas para tal fin. La medición será realizada por nutricionistas idóneos. Los datos serán codificados y digitados en un formulario en papel y luego digitalizado utilizando herramientas de gestión de bases de datos.

Ingesta alimentaria: La ingesta dietética y el patrón alimentario serán determinados mediante el método de recordatorio de 24 horas aplicado al estudiante. El recordatorio de 24 horas será levantado para tres días de la semana de los cuales se incluirá un día de fin de semana. Los datos de ingesta serán codificados y analizados utilizando la tabla de composición de alimentos para Centroamérica y Panamá del INCAP30 integrada a la herramienta informática NutrINCAP (Instituto de Nutrición de Centroamérica y Panamá, Versión 3.0, Guatemala) ${ }^{31}$. Para el recordatorio de 24 horas se utilizará un instrumento validado ${ }^{32}$ para la recolección de información referente al consumo de alimentos, macro y micronutrientes.

Valoración nutricional: La antropometría será medida en la línea de base y a las 34 semanas. Se obtendrá el peso y la talla. El análisis de los datos antropométricos será realizado por el investigador principal. Para el peso se utilizará una balanza electrónica de calibración automática marca SECA 876 (Seca Corporation Medical Scales and Measuring Systems, Hamburg, Germany) con una precisión de $100 \mathrm{~g}$. La talla será obtenida utilizando un estadiómetro marca SECA 213 (Seca Corporation Medical Scales and Measuring Systems, Hamburg, Germany) con una precisión de $1 \mathrm{~cm}$. Para el peso y la talla se le solicitará al participante utilizar ropa ligera; para la talla se solicitará mantener la posición de Frankfurt del plano horizontal ${ }^{33}$, el cual consiste en alcanzar únicamente tres puntos de contacto con el estadiómetro a la altura de los glúteos, espalda superior y cabeza. Con el peso y la talla se obtendrán los puntajes zIMCe a través del programa computacional Anthro Plus de OMS 200734. Además, se obtendrán los puntajes z de talla para la edad (zT/E) para definir retardo de crecimiento ( $\mathrm{T} T / \mathrm{E}<-2 \mathrm{DE})$. Para estimar el porcentaje de grasa corporal se empleará un plicómetro mecánico para medición de pliegues cutáneos (Lange Skinfold Caliper, modelo C-130, Cambridge, EEUU). 
Valoración de la seguridad alimentaria: Para la medición de la inseguridad alimentaria en el hogar se empleará la Escala Latinoamericana y Caribeña de Seguridad Alimentaria (ELCSA), instrumento validado para la medición de la inseguridad alimentaria en el hogar basada en experiencias vividas en los 3 meses previos a su aplicación ${ }^{28,35}$. La ELCSA mide la inseguridad alimentaria en el hogar, dimensión acceso y consumo debidos a falta de dinero o recursos ${ }^{28,36}$.

Diseño experimental: Fase 1: se realizó entre octubre 2017 a diciembre 2018, consistió en la conformación de un comité intersectorial integrado por representantes del MEDUCA, MINSA, FAO, Universidad de Panamá (UP), Universidad Interamericana de Panamá (UIP), Universidad Especializada de las Américas (UDELAS) y Dirección de las escuelas participantes. En esta fase se incluyó además la selección de las escuelas participantes. La articulación intersectorial incluye la participación de técnicos de nutrición del MEDUCA y MINSA, así como técnicos de agricultura del MIDA. Participan además técnicos de la FAO en Panamá y de la Escuela de Nutrición y Dietética de la Universidad de Panamá y de la Universidad Interamericana de Panamá. Se convocará a sesiones de trabajo para coordinar las visitas, las actividades de campo y/o modificaciones al plan de trabajo. La coordinación del proyecto será llevada por la FAO en Panamá. El financiamiento de las actividades será con fondos del programa de cooperación técnica de la FAO y del MEDUCA.

Fase 2: se realizará en abril de 2019 y consiste en el reclutamiento de los estudiantes. Para este proceso se convocará a una reunión de padres de familia con la participación de los maestros, director y personal administrativo. En la reunión se presentarán los objetivos del estudio, procedimientos a realizar, la importancia de la investigación, potenciales riesgos y beneficios de la participación en el estudio. En dicha reunión se obtendrán la forma de consentimiento informado firmado y el asentimiento de los estudiantes.

Fase 3: será el levantamiento o medición de la línea de base al inicio del año escolar del período 2019. Para la medición de la línea base se realizará una encuesta con cinco módulos que corresponden a la encuesta sociodemográfica, seguridad alimentaria, evaluación antropométrica, ingesta por recordatorio de 24 horas, y de medición de conocimiento, actitud, percepción y comportamientos sobre prácticas alimentarias saludables.

Fase 4: implementación de la intervención en las escuelas bajo tratamiento en el año escolar 2019. Durante todo el proceso se estará verificando el cumplimiento de las intervenciones propuestas: i) EAN para incrementar consumo de frutas y vegetales, lavado de manos, consumo de agua; ii) cumplimiento de la regulación de Kiosco saludable (MINSA Resolución № 49 de 2018 y MEDUCA Resolución № 3623 de 2018); y iii) realización de actividad física regularmente (3 días de la semana bajo la materia de educación física durante 2 horas clases -45 minutos c/u- bajo la supervisión de un maestro especialista).

Las actividades de EAN serán desarrolladas bajo el modelo socioecológico, el cual establece que los individuos forman parte de un entorno y éste, a su vez, debe ser abordado con acciones que faciliten la adopción de prácticas alimentarias y cambios de actitudes.

Las actuaciones de EAN incluyen un conjunto de estrategias educativas lúdicas que emplean el juego, la interacción y las experiencias para integrar conocimiento y facilitar el cambio de actitud hasta alcanzar los comportamientos deseados. Entre ellas se incluyen talleres y demostraciones de cocina empleando recetas saludables; obras de teatro y títeres sobre alimentación y estilos de vida más sanos; el uso del huerto escolar como herramienta pedagógica o laboratorio de ciencias; la elaboración de material edu-comunicacional que incluyen afiches, murales, vídeos, canciones, entre otras; así como actividades recreativas y competitivas que fomenten la práctica de actividad física diaria. Previo a la implementación de las actividades de EAN se capacitarán y dotarán de materiales a los maestros de la asignatura familia, comunidad y desarrollo y de educación física. La capacitación se realizará el primer trimestre 2019. En las capacitaciones a los maestros se incluirán a directores y personal administrativo de los colegios.

Fase 5: corresponderá a la evaluación final, después de 34 semanas de intervención. Durante noviembre y diciembre 2019. Se incluye la realización de los siguientes módulos: i) antropometría; ii) recordatorio de 24 horas para determinar ingesta dietética y patrón alimentario; iii) conocimiento, actitud, percepción y comportamiento; y, iv) seguridad alimentaria.

\section{Consideraciones éticas}

El protocolo de estudio fue revisado y aprobado por el Comité de Bioética de la Universidad de Panamá (CBUP) (Referencia № CBUP/075/2019). Además, se registró en la Coordinación de Regulación de la investigación de la Dirección General de Salud (DIGESA) del MINSA (Referencia Registro Protocolos MINSA/DIGESA/№ 881). El protocolo fue registrado en la plataforma internacional de ensayos № ISRCTN28920505. Adicionalmente, previo a una inducción sobre el propósito del estudio, procedimientos, beneficios y potenciales riesgos, se solicitará a cada acudiente de los 
participantes la firma del consentimiento informado, así como la firma del asentimiento por parte del estudiante.

\section{Plan de análisis estadísticos}

Todos los análisis estadísticos se realizarán con el paquete informático Stata 12.0 (Stata, College Station, Texas USA). Las variables continuas serán presentadas como media y desviación estándar cuando sigan una distribución normal, o como mediana y rango intercuartiles cuando presenten una distribución asimétrica, posterior a la prueba de normalidad de Shapiro Wilks. Las variables categóricas serán presentadas como frecuencia, porcentaje e intervalo de confianza 95\% (IC95\%). Para comparar el efecto del programa Escuela Saludable versus no saludable sobre la proporción de sobrepeso y obesidad se empleará una prueba de proporciones. Las variables continuas, alimentarias y de estado nutricional serán comparadas utilizando estadística paramétrica o no paramétrica según el tipo de distribución de los datos, pruebas t de Student o Wilcoxon Mann Whitney. Para las variables categóricas se utilizarán las pruebas $\mathrm{Chi}^{2}$ o de proporciones. Para comparar el cambio antes y después de la intervención se empleará la prueba no paramétrica McNemar. Por último, para determinar la magnitud de la asociación entre la proporción de sobrepeso y obesidad debido a la intervención, se utilizará modelamiento multivariado a través de regresión logística ajustando por variables sociodemográficas, de actitud, comportamentales, alimentarias, por los efectos fijos de las escuelas y por errores estándares robustos. El modelo de regresión será seleccionado a través del método paso a paso, incluyendo variables con una probabilidad superior a 0,2 . Se considerará significancia estadística cuando los valores $\mathrm{p}$ asociados a las pruebas estadísticas sean menores de 0,05 .

\section{DIscusıón}

Panamá ha logrado significativos avances en la lucha contra el hambre y la malnutrición en todas sus formas. Prueba de lo anterior fue lo reportado en el Panorama de la Inseguridad Alimentaria de América Latina y el Caribe (ALC) 2015 de la Organización de las Naciones Unidas para la Alimentación y la Agricultura (FAO), en donde se reportó que la prevalencia de subalimentación a nivel nacional se redujo de $26,4 \%$ a $9,5 \%$ en los períodos $1990-1992$ y 2014-2016, respectivamente ${ }^{37}$. De acuerdo al último Panorama de la Seguridad Alimentaria y Nutricional (2018), en el país aún existen poco más de 400 mil personas que no logran cubrir sus demandas nutricionales ${ }^{38}$. Sin embargo, las otras caras de la malnutrición persisten como problemas de salud pública en el país.

Son varios los compromisos asumidos por el Gobierno panameño en la lucha contra el hambre y la malnutrición. En septiembre 2015, asumió al más alto nivel político el reto global de erradicar el hambre y la pobreza al 2030 establecido en los Objetivos de Desarrollo Sostenible (ODS) mediante el Decreto Ejecutivo № 393 (Gaceta Oficial № 27870-B, 15 de septiembre de 2015), así como también con la creación de la Comisión Interinstitucional de Apoyo y Seguimiento a la implementación de los ODS $^{39}$.

El país ha emprendido acciones de lucha contra la malnutrición, entre las que se encuentran la implementación del Plan Nacional para la Seguridad Alimentaria y Nutricional (SAN) 2017-2021; la adopción y compromiso con los lineamientos en materia de Seguridad Alimentaria y Nutricional del Plan SAN de la Comunidad de Estados Latinoamericanos y Caribeños (CELAC) al $2025^{40}$. Más recientemente, en el país se ha puesto en marcha la Ley 75 de 2017 que establece la obligatoriedad de los ambientes alimentarios saludables en las escuelas ${ }^{41}$. Asimismo, se han implementado dos regulaciones sobre kioscos escolares que prohíben la venta de alimentos chatarra en las escuelas, la Resolución MINSA № 49 de 2018 y la Resolución MEDUCA № 3623 de 2018.

Pese a ello, la malnutrición en todas sus formas afecta a las comunidades más vulnerables del país. Panamá no sólo enfrenta los problemas relacionados con las deficiencias nutricionales, sino que cada vez es más preocupante los problemas relacionados con el sobrepeso y la obesidad en todos los grupos etarios y sus terribles consecuencias, que a largo plazo son causadas por esta forma de malnutrición ${ }^{42-44}$. Asimismo, en el país aún persisten problemas relacionados con el retardo de crecimiento ${ }^{45}$. En escolares, el sobrepeso representa un creciente problema de salud pública en Panamá1, lo cual pone en riesgo el alcance de las metas de desarrollo sostenibles. Paralelamente, datos más recientes del Monitoreo del Estado Nutricional (MONINUT), realizado por el MINSA en 2014 y 2017, revelan que la prevalencia de sobrepeso en escolares a nivel nacional alcanza al $29 \%$ y $29,9 \%$, respectivamente 2 .

Según la Organización Mundial de la Salud (OMS), el sobrepeso y la obesidad se definen como la acumulación excesiva de tejido graso corporal, que representa serios problemas de salud al individuo y que resulta de un desequilibrio entre las calorías consumidas y gastadas ${ }^{46}$. La obesidad incrementa el riesgo de desarrollar ENT, las que se ubican entre 
las 10 primeras causas de muerte en el país ${ }^{47}$. Lo anterior plantea la necesidad urgente de acciones por parte del Gobierno Panameño en atacar el creciente problema de sobrepeso y obesidad en la edad escolar.

Por otra parte, los ambientes alimentarios y el sistema alimentario actual son probablemente los mayores causantes de las alarmantes cifras de malnutrición en el país ${ }^{48}$. Haciendo necesario implementar sistemas de vigilancia nutricional, e identificación, implementación y evaluación de políticas públicas multisectoriales basadas en evidencia para abordar este problema, así como también determinando el impacto de intervenciones en ambientes escolares sobre el IMC, la obesidad y la conducta alimentaria.

Por lo tanto, las intervenciones sobre el ambiente alimentario, a nivel de los hogares y en las escuelas, pudieran tener impacto en la reducción y prevención del sobrepeso y la obesidad en escolares ${ }^{49,50}$. Es así que, la escuela con atención a sus múltiples intervenciones es la mejor estrategia para promover ambientes alimentarios saludables. Varios autores han reportado efecto de intervenciones de educación nutricional sobre marcadores antropométricos, alimentarios y conductuales $24,51-55$, pero con resultados heterogéneos en ensayos controlados y no en el marco de políticas públicas. Se propone entonces un modelo que sustenta una intervención socioecológica, la cual combina la promoción de alimentación sana, la seguridad alimentaria, el consumo de agua, la realización diaria de actividad física con mediciones antropométricas y sociodemográficas, fomentando actitudes que mejorarán los hábitos alimenticios, y a futuro, un comportamiento alimentario sano y autoregulado; enmarcado en el contexto social panameño.

Para implementar políticas y programas contra la obesidad es imprescindible aumentar la coordinación y la articulación multisectorial, incorporando a todos los actores vinculados a la seguridad alimentaria y nutricional, desde instituciones públicas, sociedad civil, sector privado, academia, productores y consumidores, entre otros, con el fin de transformar los actuales sistemas alimentarios para hacerlos más sostenibles, justos e inclusivos. En este sentido, y a pesar de los progresos descritos en los párrafos precedentes, se constata aún vacíos importantes en el diseño e implementación de políticas públicas intersectoriales que cuenten con la capacidad de modificar sustantivamente los ambientes alimentarios en entornos escolares. Los vacíos incluyen la falta de un sistema de vigilancia nutricional; desarrollo de materiales y acciones de EAN; herramientas para la adecuada implementación de la regulación de los kioscos y cafeterías escolares, así como la promoción de la actividad física regular, como también un enfoque intersectorial y territorial con participación de actores clave, todos estos elementos parte de un ambiente escolar y alimentario saludable.

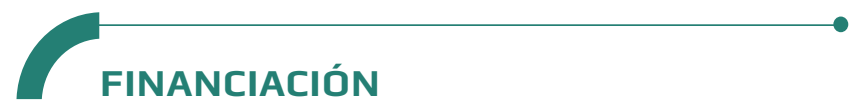

El estudio es financiado mediante el proyecto TCP/ PAN/3606 "Diseño de una Política Pública Multisectorial contra el Sobrepeso y la Obesidad en Escolares de Educación Primaria" de la Organización de las Naciones Unidas para la Alimentación y la Agricultura (FAO) en Panamá. Se agradece además a la Agencia Mexicana de Cooperación Internacional (AMEXID) por el apoyo financiero parcial para la fase de campo mediante el Programa Mesoamérica Sin Hambre.

Las opiniones y conclusiones del estudio son responsabilidad de sus autores y no representan la posición de la FAO.

\section{$\longrightarrow$ C CONFLICTO DE INTERESES}

Los autores expresan que no existen conflictos de interés al redactar el manuscrito.

\section{REFERENCIAS}

(1) Ministerio de Economía y Finanzas, Instituto Nacional de Estadísticas y Censos. Encuesta de Niveles de Vida 2008 [Internet]. Panama; 2008. Available from: http://www. contraloria.gob.pa/inec/Aplicaciones/ENV2008/intro.html

(2) Ministerio de Salud de Panamá. Monitoreo Nutricional en las Instalaciones de Salud del MINSA (MONINUT). Panamá; 2014.

(3) Nicklas T, O'Neil CE, Stuff JE, Hughes SO, Liu Y. Global Childhood Obesity Update. Child Obes. 2011; 7(2): 143-6.

(4) Tzioumis E, Adair LS. Childhood dual burden of under- and overnutrition in low- and middle-income countries: A critical review. Food Nutr Bull. 2014; 35(2): 230-43.

(5) Webber L, Kilpi F, Marsh T, Rtveladze K, Brown M, McPherson $K$. High rates of obesity and non-communicable diseases predicted across Latin America. PLoS One. 2012; 7(8): e39589.

(6) Shrimpton R, Rokx C. The Double Burden of Malnutrition: A Review of Global Evidence. HNP Discussion Paper: The World Bank. 2012.

(7) Rivera J, Barquera S, Campirano F, Campos I, Safdie M, Tovar V. Epidemiological and nutritional transition in Mexico: rapid increase of non-communicable chronic diseases and obesity. Public Health Nutr. 2002; 5(1a): 113-22. 
(8) Black RE, Victora CG, Walker SP, Bhutta ZA, Christian P, De Onis $M$, et al. Maternal and child undernutrition and overweight in low-income and middle-income countries. Lancet. 2013; 382(9890): 427-51.

(9) Lawlor DA, Jago R, Noble SM, Chittleborough CR, Campbell $R$, Mytton J, et al. The Active for Life Year 5 (AFLY5) school based cluster randomised controlled trial: study protocol for a randomized controlled trial. Trials. 2011.

(10) Morales-Ruán M del C, Shamah-Levy T, Amaya-Castellanos Cl, Salazar-Coronel AA, Jiménez-Aguilar A, Amaya-Castellanos $M A$, et al. Effects of an intervention strategy for school children aimed at reducing overweight and obesity within the state of Mexico. Salud Publica Mex. 2014; 56(2): S113-22.

(11) Alaimo K, Oleksyk SC, Drzal NB, Golzynski DL, Lucarelli JF, Wen $Y$, et al. Effects of changes in lunch-time competitive foods, nutrition practices, and nutrition policies on low-income middle-school children's diets. Child Obes. 2013; 9(6): 509-23.

(12) Jomaa LH, McDonnell E, Probart C. School feeding programs in developing countries: Impacts on children's health and educational outcomes. Nutr Rev. 2011; 69(2): 83-98.

(13) Bustos N, Olivares S, Leyton B, Cano M, Albala C. Impact of a school-based intervention on nutritional education and physical activity in primary public schools in Chile (KIND) programme study protocol: cluster randomised controlled trial. BMC Public Health. 2016; 16(1217): 1-11.

(14) Ministerio de Salud de Panamá. Política Nacional de Salud y Lineamientos Estratégicos 2016 - 2025. Panamá: Ministerio de Salud de Panamá; 2015. 113 p.

(15) Farro K, Montero I, Vergara E, Ríos-Castillo I. Elevado consumo de azúcares y grasas en niños de edad preescolar de Panamá: Estudio transversal. Rev Chil Nutr. 2018; 45(1): 7-16.

(16) Hernández M, Núñez G. Consumo y verificación del contenido de sodio y grasa saturada y grasa total de productos ultra procesados por los escolares de dos colegios públicos del país. Universidad de Panamá; 2016.

(17) Bradford B, Berg S, Hickson C. The Untapped Potential of Physical and Health Education. Phys Heal Educ J. 2016; 81(3): 1-12.

(18) Contento I R. Nutrition education: linking research, theory, and practice. Asia Pac J Clin Nutr. 2008; 17(S1): 176-9.

(19) Carroll J, Epel ES, Puska P, Sinclair K, Meneghini L, Hall PA, et al. Theories of Behavior Change. In: Encyclopedia of Behavioral Medicine. 2012. p. 1963-1963.

(20) Prochaska JO, Velicer WF. The transtheoretical model of health behavior change. Am J Heal Promot. 1997; 12(1): 38-48.

(21) Johnson R, Stewart D. The International Encyclopedia of the Social \& Behavioral Sciences. Behav Soc Sci Librar. 2002; 21(2): 73-83.

(22) Coleman KJ, Shordon M, Caparosa SL, Pomichowski ME, Dzewaltowski DA. The healthy options for nutrition environments in schools (Healthy ONES) group randomized trial: using implementation models to change nutrition policy and environments in low income schools. Int ] Behav Nutr Phys Act. 2012; 9(1): 80.

(23) Contento I, Balch G, Bronner Y, Lytle L, Maloney S, Olson C, et al. The effectiveness of nutrition education and implications for nutrition education policy, programs, and research: a review of research. J Nutr Educ. 1995; 27(6): 277-418.

(24) Bogart LM, Cowgill BO, Elliott MN, Klein DJ, Hawes-Dawson J, Uyeda K, et al. A randomized controlled trial of Students for Nutrition and exercise: A community-based participatory research study. J Adolesc Health. 2014; 55(3): 415-22.

(25) Antes G. The new CONSORT statement. BMJ. 2010; 340(8): c1432.

(26) Ministerio de Salud de Panamá. Guías Alimentarias para Panamá. 2013. 19 p.

(27) Rios-Castillo I, Farro K, Escala L, Gonzalez E, Lebrija A. Validity and Reliability of Knowledge, Attitude, Perception and Behaviors on Healthy Feeding in School-Age Children in Panama. J Nutr Educ Behav. 2019; 51(7): S119-S120.

(28) FAO. Escala Latinoamericana y Caribeña de Seguridad Alimentaria (ELCSA): Manual de uso y aplicaciones. FAO. Roma; 2012. $78 \mathrm{p}$.

(29) FAO. Guía para medir la diversidad alimentaria a nivel individual y del hogar. Rome, Italy; 2013. 58 p.

(30) Instituto de Nutrición de Centroamérica y Panamá. Tabla de composición de alimentos. Segunda edición. Menchú MT, Méndez H, editors. Guatemala; 2012. 137 p.

(31) Instituto de Nutrición de Centroamérica y Panamá. Software NutrINCAP [Internet]. Instituto de Nutricion de Centroamérica y Panamá (INCAP). 2014. Available from: http://www.incap. int/index.php/es/servicios/software-nutrincap

(32) Baranowski T. 24-Hour Recall and Diet Record Methods. In: Nutritional Epidemiology. 2013.

(33) CDC. National Health and Nutrition Examination Survey III: Body Measurements (Anthropometry) [Internet]. Rockville, Centers for Disease Control and Prevention; 1988. 62 p. Available from: https://wwwn.cdc.gov/nchs/data/nhanes3/ manuals/anthro.pdf

(34) WHO. WHO AnthroPlus software [Internet]. Growth reference 5-19 years. 2007. Available from: http://www.who.int/ growthref/tools/en/

(35) Marques ES, Reichenheim ME, de Moraes CL, Antunes MML, Salles-Costa R. Household food insecurity: a systematic review of the measuring instruments used in epidemiological studies. Public Health Nutr. 2015; 18(5): 877-92.

(36) De Haro-Mota R, Marceleño-Flores S, Bojórquez-Serrano Jl, Nájera-González O, Haro-Mota R de, Marceleño-Flores S, et al. La inseguridad alimentaria en el estado de Nayarit, México, y su asociación con factores socioeconómicos. Salud Publica Mex. 2016; 58(4): 421-27.

(37) FAO. Panorama de la Inseguridad Alimentaria en América Latina y el Caribe. La Región alcanza las metas internacionales del hambre. 2015. $78 \mathrm{p}$.

(38) FAO, OPS/OMS, UNICEF, WFP. Panorama de la Seguridad Alimentaria y Nutricional en América Latina y el Caribe: Desigualdad y Sistemas Alimentarios. Santiago; 2018. 133 p.

(39) United Nations. SDG 2: End hunger, achieve food security and improved nutrition, and promote sustainable agriculture [Internet]. Sustainable Development Knowledge Platform. 2014. Available from: https://sustainabledevelopment.un.org

(40) CELAC. The CELAC Plan for Food and Nutrition Security and the Eradication of Hunger 2025. 2016. 10 p.

(41) Gobierno de la República de Panamá. Ley N 75 de 2017 Que establece medidas para promover la alimentación adecuada y estilo de vida saludable en los centros educativos [Internet]. 28406 Panama: Gaceta Oficial; 2017 p. 36. Available from: https://www.gacetaoficial.gob.pa/pdfTemp/28406/ GacetaNo_28406_20171115.pdf 
(42) Mc Donald A, Bradshaw RA, Fontes F, Mendoza EA, Motta JA, Cumbrera $A$, et al. Prevalence of obesity in panama: some risk factors and associated diseases. BMC Public Health. 2015; 15(1): 1075.

(43) Gordon C. Transición epidemiológica y las diferencias en la salud de la población entre la periferia y el centro urbano del Área Metropolitana de Panamá, 2001- 2011. Invest Pens Crit. 2015; 3(1): 17-38.

(44) Mujica-Coopman MF, Brito A, López de Romaña D, Ríos-Castillo I, Cori H, Olivares M. Prevalence of Anemia in Latin America and the Caribbean. Food Nutr Bull. 2015; 36(S2): S119-28.

(45) Ministerio de Salud de Panamá. VII Censo de Talla de Escolares de Primer Grado de las Escuelas Oficiales de Panamá 2013. Panama; 2013.

(46) WHO. Global Health Observatory (GHO) data [Internet]. 2014 [cited 2018 Feb 19]. Available from: http://www.who.int/gho/ en/

(47) Instituto Nacional de Estadísticas y Censos. Estadísticas Vitales - Volumen III - Defunciones [Internet]. Situación Demográfica. Panamá; 2017. Available from: https://www.contraloria.gob. $\mathrm{pa/INEC/Publicaciones/}$

(48) Afshin A, Schutte AE, Forouzanfar MH, Reitsma MB, Sur P. Health Effects of Overweight and Obesity in 195 Countries over 25 Years. N Engl J Med. 2017; 377(1): 13-27.

(49) Cooke L. The importance of exposure for healthy eating in childhood: A review. J Hum Nutr Diet. 2007; 20(4): 294-301.
(50) Robinson-O'Brien R, Story M, Heim S. Impact of garden-based youth nutrition intervention programs: a review. J Am Diet Assoc. 2009; 109(2): 273-80.

(51) Briones-Villalba R, Briones-Villalba RA, Gómez-Miranda LM, Ortiz-Ortiz M, Rentería I. Efecto de un programa de actividad física y educación nutricional para reducir el consumo de bebidas azucaradas y desarrollo de la obesidad en escolares de Tijuana, México. Rev Esp Nutr Hum Diet. 2018; 22(3): 235-42.

(52) Vio F, Salinas J, Montenegro E, González CG, Lera L. Efecto de una intervención educativa en alimentación saludable en profesores y niños preescolares y escolares de la región de Valparaíso, Chile. Nutr Hosp. 2014; 29(6): 1298-304.

(53) Bogart LM, Elliott MN, Uyeda K, Hawes-Dawson J, Klein DJ, Schuster MA. Preliminary healthy eating outcomes of SNaX, a pilot community-based intervention for adolescents. J Adolesc Health. 2011; 48(2): 196-202.

(54) Kain B], Uauy DR, Leyton DB, Cerda RR, Olivares CS, Vio DF. Efectividad de una intervención en educación alimentaria y actividad física para prevenir obesidad en escolares de la ciudad de Casablanca, Chile (2003-2004). Rev Med Chile. 2008; 136(1): 22-30.

(55) Fernández LLL, Dinamarca BL, Bercovich JK, del Río FV. Evaluación de una intervención educativa para la prevención de la obesidad infantil en escuelas básicas de Chile. Nutr Hosp. 2013; 28(4): 1156-64. 